\title{
UNSTEADY MHD BOUNDARY LAYER FLOW OF AN UPPER CONVECTED MAXWELL FLUID PAST A STRETCHING SHEET WITH FIRST ORDER CONSTRUCTIVE/DESTRUCTIVE CHEMICAL REACTION
}

\author{
Swati Mukhopadhyay and Rama Subba Reddy Gorla ${ }^{2}$ \\ ${ }^{1}$ Department of Mathematics, The University of Burdwan, Burdwan-713104, W. B., India, \\ Email: swati bumath@yahoo.co.in \\ ${ }^{2}$ Department of Mechanical Engineering, Cleveland State University, Cleveland, OH 44115, USA, \\ Email: $\underline{\text { r.gorla@csuohio.edu }}$
}

\begin{abstract}
:
The mass transfer of unsteady two-dimensional flow of MHD non-Newtonian Maxwell fluid over a stretching surface in the presence of first order constructive/destructive chemical reaction is presented. Upper convected Maxwell (UCM) fluid model is used here to characterize the nonNewtonian behavior of the fluid. Using similarity solutions the governing partial differential equations are transformed to ordinary differential equations and are then solved numerically by shooting method. The flow field and mass transfer are significantly influenced by the governing parameters. The results show that fluid velocity initially decreases with increasing unsteadiness parameter ( 0 to 0.3$)$ and concentration decreases significantly due to unsteadiness. The effect of increasing values of the Maxwell parameter (O to 0.4) is to suppress the velocity field. The concentration is enhanced with increasing Maxwell parameter. The fluid velocity decreases with increasing magnetic parameter (0 to 0.3 ).
\end{abstract}

Keywords: Unsteady flow, MHD, non-Newtonian Maxwell fluid, stretching sheet, chemical reaction, boundary layer flow.

\section{NOMENCLATURE:}

$B_{0}$ magnetic field strength (T)

$C$ concentration of the species of the fluid $\left(\mathrm{kg} \mathrm{m}^{-3}\right)$

$C_{w}$ concentration of the wall of the surface $\left(\mathrm{kg} \mathrm{m}^{-3}\right)$

$C_{\infty}$ free-stream concentration $\left(\mathrm{kg} \mathrm{m}^{-3}\right)$

$D$ diffusion coefficient of the diffusing species $\left(\mathrm{m}^{2} \mathrm{~s}^{-1}\right)$

$f$ non-dimensional stream function (-)

$\mathrm{Ha}$ magnetic parameter (-)

$k_{1}$ reaction rate $\left(\mathrm{s}^{-1}\right)$

$M$ unsteadiness parameter (-)

Sc Schmidt number (-)

$u, v$ components of velocity in $\mathrm{x}$ and $\mathrm{y}$ directions $\left(\mathrm{m} \mathrm{s}^{-1}\right)$
Greek symbols:

$\beta$ Maxwell parameter (-)

$\eta$ similarity variable (-)

$\gamma$ reaction rate parameter (-)

$\lambda$ relaxation time $\left(\mathrm{s}^{-1}\right)$

$\nu$ kinematic viscosity $\left(\mathrm{m}^{2} \mathrm{~s}^{-1}\right)$

$\rho$ fluid density $\left(\mathrm{kg} \mathrm{m}^{-3}\right)$

$\sigma$ electrical conductivity $\left(\mathrm{S} \mathrm{m}^{-1}\right)$

$\psi$ stream function $\left(\mathrm{m}^{2} \mathrm{~s}^{-1}\right)$

$\phi$ non-dimensional concentration (-)

\section{Introduction}

In the recent years, the boundary layer flows of non-Newtonian fluids have been given considerable attention due to ever increasing engineering applications particularly in polymer depolarization, bubble columns, fermentation, composite processing, boiling, plastic foam processing, bubble absorption and many others. Moreover, non-Newtonian fluid flows generated by a stretching sheet have been widely analyzed for the importance in several manufacturing processes such as extrusion of molten polymers through a slit die for the production of plastic sheets, processing of food stuffs, paper production, and wire and fiber coating (Hayat et al., 2012a). The fluid employed in material processing or protective castings are generally non-Newtonian 
(example, most of the paints). The governing equations of non-Newtonian fluids are highly non-linear and much more complicated than those of Newtonian fluids. Therefore, the flow behaviour of non-Newtonian fluids is quite different from that of Newtonian fluids. An assessment of technological applications requires a knowledge of the rheological characteristics of the non-Newtonian fluids. For this, investigations on nonNewtonian fluid are necessary to obtain a thorough idea about them (Mukhopadhyay, 2012a). Due to complexity of these fluids, no single constitutive equation exhibiting all properties of such fluids is available. Several models can be found in this regard. The vast majority of non-Newtonian fluid models are concerned with simple models like the power law and grade two or three (Hassanien, 1996), Sadeghy and Sharifi (2004), Serdar and Salih Dokuz (2006), Haroun (2007), Siddiqui et al. (2008), Abel et al. (2008), Sajid et al. (2009)]. Though these models are very simple but deviate much from reality in results. Because in most industrially important processes, one is often encountered with flows occurring at high Deborah numbers. A second-grade model is not expected to render meaningful results when dealing with fast flows of highly elastic fluids such as polymer melts. There are also some serious concerns about the soundness of the second-grade model from a thermodynamics point of view, and it has been argued that this model, in its current form, suffers from several anomalous features. In modelling fluids with shear-dependent viscosity, power-law model has a vast usage. As this model deals with a simple non-linear equation of state for inelastic fluids, it fails to predict the effects of elasticity. The fluids of grade two or three can calculate the effects of elasticity. But in these models, the viscosity is not shear dependent as the (shear) viscosity is constant and also they are unable to predict the effects of stress relaxation. The simplest model for the rheological effects of viscoelastic fluids is the upper-convected Maxwell (UCM) fluid where the dimensionless relaxation time is small. Upper-convected Maxwell (UCM) fluid, a subclass of rate type fluids, can predict the stress relaxation but it does not exhibit the normal stress effects and shear thinning/shear thickening (Hayat et al. (2012b)]. Therefore, this model became more popular. In this model, as the complicating effects of shear-dependent viscosity have been excluded one can focus solely on the effects of a fluid's elasticity on the characteristics of its boundary layer (Heyhat and Khabazi (2010)]. But the 'Upper convected Maxwell model' lacks in describing the typical relation between shear rate and shear stress in a simple shear flow of a real fluid: it predicts only a linear relation (Mukhopadhyay (2012b)]. The Maxwell model uses convected derivatives instead of time derivatives (Turkyilmazoglu and Pop (2013)].

Recently, the subject of hydromagnetics has attracted the attention of many authors due to its applications to problems which have geophysical and astrophysical significance. In modern metallurgical and metal-working processes, the study of MHD flow of an electrically conducting fluid is of considerable interest (Mukhopadhyay (2012c)]. To purify the molten metals from non-metallic inclusions, hydromagnetic techniques are used. This type of problem is very much useful to polymer technology and metallurgy. Hayat et al. (2006) reported a series solution for MHD boundary layer flow of an upper convected Maxwell fluid over a porous stretching sheet. In another paper, Hayat et al. (2008) constructed analytic solutions for unsteady MHD flow in a rotating Maxwell fluid through a porous medium. Analytic solution of the governing flow problem was obtained by means of the Fourier sine transform. Mukhopadhyay (2011) reported the magnetic effects on unsteady non-isothermal stretching sheet assuming the temperature variation with space and also with time. In another paper, Mukhopadhyay (2012d) investigated the combined effects of MHD and partial slip on flow over an exponentially stretching sheet. In this paper, the author also considered the effects of suction/blowing and thermal radiation. Recently, Bhattacharyya et al. (2013) discussed the MHD boundary layer flow for diffusion of a chemically reactive species. In this paper, they also analyzed the combined effects of unsteadiness and suction/blowing in presence of first order constructive/destructive chemical reaction.

Mass transfer phenomenon is used in various scientific disciplines for different systems and mechanisms that involve molecular and convective transport of atoms and molecules. The driving force for mass transfer is the difference between the concentration (Hayat et al. (2011)]. The transport of mass and momentum with chemical reactive species in the flow caused by a linear stretching sheet is discussed by Andersson et al. (1994). Cortell (2007) discussed mass transfer with chemically reactive species for two classes of viscoelastic fluid over a porous stretching sheet. Of late, Mukhopadhyay and Bhattacharyya (2012) discussed the mass transfer effects on Maxwell fluid flow past an unsteady stretching sheet.

In most of the studies mentioned above, the flow, temperature and concentration fields are considered to be at steady state. However, due to a sudden stretching of the flat sheet or by a step change of the temperature of the sheet etc. heat, and mass transfer can be unsteady. The inviscid flow is developed instantaneously when the surface is impulsively stretched with certain velocity. However, near the sheet the flow in the viscous layer is developed slowly and it becomes a fully developed steady flow after a certain instant of time. Several researchers viz. Andersson et al. (2000), Dandapat et al. (2003, 2007), Ali and Magyari (2007) studied the problem for unsteady stretching surface under different conditions by using a similarity method to transform governing time-dependent boundary layer equations into a set of ordinary differential equations. Among these Unsteady MHD boundary layer flow of an upper convected Maxwell fluid past a stretching sheet..... 
studies, Dandapat et al. $(2003,2007)$ investigated the effect of the thermocapillarity on the hydrodynamics and heat transfer in case of a liquid film on a stretching surface and showed that thermocapillarity induces surfacetension gradients along the horizontal interface between the passive gas and the liquid film. Further, Elbashbeshy and Bazid (2004) have presented similarity solutions of the boundary layer equations, that describe the unsteady flow and heat transfer over an unsteady stretching sheet. Sharidan et al. (2006) analysed the unsteady flow and heat transfer over a stretching sheet in a viscous and incompressible fluid by considering both the variable wall temperature (VWT) and variable heat flux (VHF) conditions. Tsai et al. (2008) obtained solutions for unsteady flow and heat transfer over a stretching sheet and reported the effects of variable heat source/sink on heat transfer characteristics. Mukhopadhyay (2009) obtained similarity solutions for unsteady mixed convection flow past a stretching sheet considering the combined effects of porous medium and thermal radiation. In that paper the variable permeability was assumed. Chamkha et al. (2010) discussed the effects of chemical reaction on unsteady free convective flow over a porous stretching sheet embedded in a porous medium. Assuming the fluid properties to be constant, a first order homogeneous chemical reaction was assumed in their model. They obtained the numerical solution by using an efficient tri-diagonal implicit finitedifference method.

No attempt has been made so far to analyze the MHD upper convected Maxwell fluid flow and mass transfer past an unsteady stretching surface in presence of first order constructive/destructive chemical reaction. The present work aims to fill the gap in the existing literature. An attempt is made to study the non-Newtonian Maxwell fluid and mass transfer in presence of first order constructive/destructive chemical reaction. Similarity solutions are obtained and the reduced ordinary differential equations are solved numerically using shooting method. The effects of governing parameters on velocity and concentration fields are investigated and analysed with the help of their graphical representations.

\section{Equations of Motion}

We consider laminar boundary-layer two-dimensional flow and mass transfer of an incompressible electrically conducting non-Newtonian Maxwell fluid (in presence of a transverse magnetic field) over an unsteady stretching sheet (Fig.1). The flow is permeated by a non-uniform magnetic field of strength $B_{1}=\frac{B_{0}}{\sqrt{1-\alpha t}}$ applied normal to the surface, $B_{0}$ is the initial strength of the magnetic field. Let $C_{w}$ be the concentration at the sheet and concentration far away from the sheet is $C_{\infty}$. Also the reaction of species be the first order homogeneous chemical reaction of rate constant $k_{1}$ (Hayat et al. (2011)].

The unsteady fluid and mass flows start at $t=0$. The sheet emerges out of a slit at origin $(x=0, y=0)$ and moves with non-uniform velocity $U(x, t)=\frac{b x}{1-\alpha t}$ where $b, \alpha$ are positive constants both having dimensions (time $^{-1}, b$ is the initial stretching rate and $\frac{b}{1-\alpha t}$ is the effective stretching rate which is increasing with time. In case of polymer extrusion, the material properties of the extruded sheet may vary with time.

The governing equations of such type of flow are, in the usual notation (Alizadeh-Pahlavan and Sadeghy, 2009),

$$
\begin{aligned}
& \frac{\partial u}{\partial x}+\frac{\partial v}{\partial y}=0, \\
& \frac{\partial u}{\partial t}+u \frac{\partial u}{\partial x}+v \frac{\partial u}{\partial y}+\lambda\left(u^{2} \frac{\partial^{2} u}{\partial x^{2}}+v^{2} \frac{\partial^{2} u}{\partial y^{2}}+2 u v \frac{\partial^{2} u}{\partial x \partial y}\right)=v \frac{\partial^{2} u}{\partial y^{2}}-\frac{\sigma B_{1}^{2}}{\rho}\left(u+\lambda v \frac{\partial u}{\partial y}\right), \\
& \frac{\partial C}{\partial t}+u \frac{\partial C}{\partial x}+v \frac{\partial C}{\partial y}=D \frac{\partial^{2} C}{\partial y^{2}}-k_{1}\left(C-C_{\infty}\right) .
\end{aligned}
$$

For the Upper Convected Maxwell (UCM) fluid model used in this paper, no unsteady terms for the shear stress term need to be included as mentioned by Alizadeh-Pahlavan and Sadeghy (2009). Therefore, no unsteady terms appear to the coefficient of $\lambda$. 


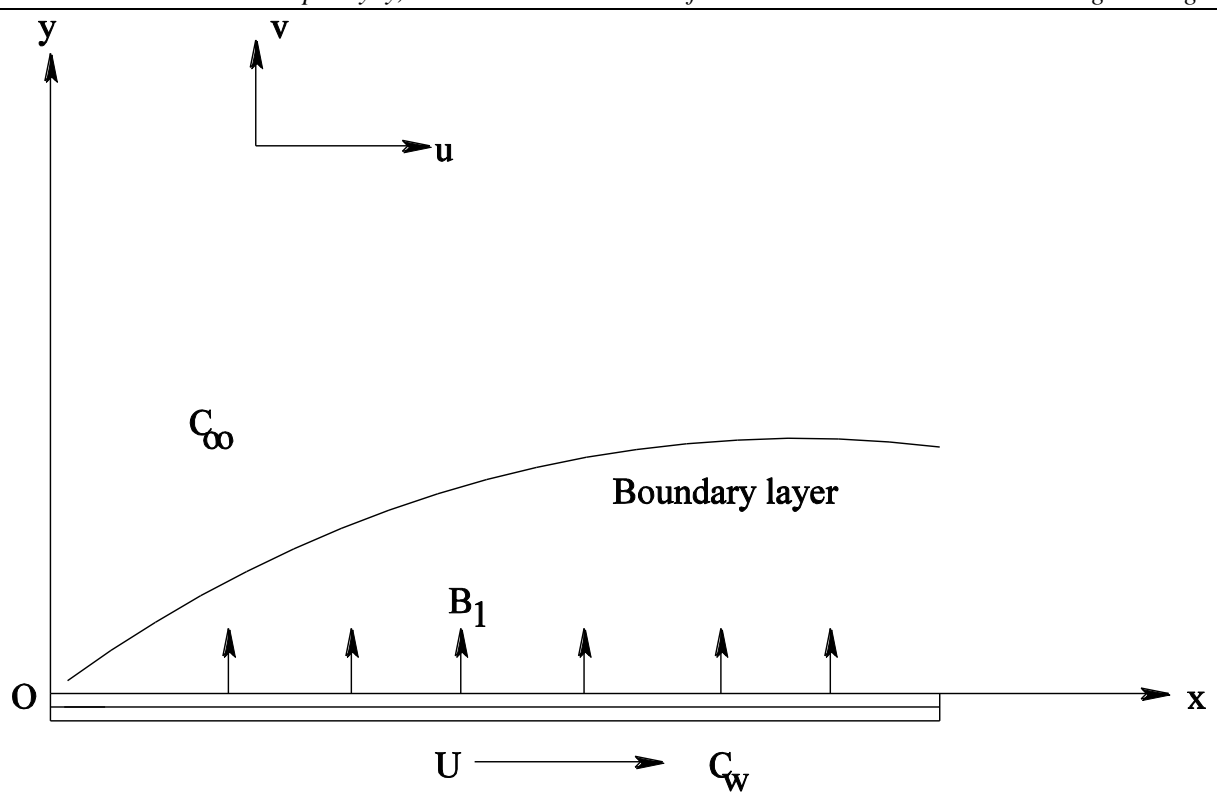

Fig. 1: Sketch of the physical problem.

Here $u$ and $v$ are the components of velocity respectively in the $x$ and $y$ directions, $\rho$ is the fluid density, where $v$ is the kinematic viscosity of the fluid, $\sigma$ is the electrical conductivity of the fluid, $C$ is the concentration of the species of the fluid, $D$ is the diffusion coefficient of the diffusing species in the fluid, $k_{1}(t)=\frac{k_{0}}{1-\alpha t}$ is the time-dependent reaction rate; $k_{1}>0$ stands for destructive reaction whereas $k_{1}<0$ stands for constructive reaction, $k_{0}$ is a constant, $\lambda=\lambda_{0}(1-\alpha t)$ is the relaxation time of the period, $\lambda_{0}$ is a constant.

\subsection{Boundary conditions}

The appropriate boundary conditions for the problem are given by

$$
\begin{aligned}
& u=U(x, t), v=0, C=C_{w}(x, t) \text { at } y=0, \\
& u \rightarrow 0, C \rightarrow C_{\infty} \text { as } \mathrm{y} \rightarrow \infty .
\end{aligned}
$$

where the concentration of the surface of the sheet is similarly assumed to vary both along the sheet and with time, in accordance with $C_{w}(x, t)=C_{\infty}+b x(1-\alpha t)^{-2}$.If $b$ is positive (negative) in proportion to $x$, the wall concentration $C_{w}(x, t)$ increases (reduces).Moreover, the amount of increase (reduction) in concentration along the sheet increases with time. The expressions for $U(x, t), C_{w}(x, t), \lambda(t), k_{1}(t)$ are valid for time $t<\alpha^{-1}$.

\subsection{Method of solution}

We now introduce the following relations for $u, v$ and $\phi$ as

$u=\frac{\partial \psi}{\partial y}, v=-\frac{\partial \psi}{\partial x}$ and $\phi=\frac{C-C_{\infty}}{C_{w}-C_{\infty}}$

where $\psi$ is the stream function.

We introduce $\eta=\sqrt{\frac{c}{v(1-\alpha t)}} y, \psi=\sqrt{\frac{v c}{(1-\alpha t)}} x f(\eta), C=C_{\infty}+b x(1-\alpha t)^{-2} \phi(\eta)$.

With the help of the above relations, the governing equations finally reduce to 
$M\left(\frac{\eta}{2} f^{\prime \prime}+f^{\prime}\right)+f^{\prime 2}-f f^{\prime \prime}+\beta\left(f^{2} f^{\prime \prime \prime}-2 f f^{\prime} f^{\prime \prime}\right)=f^{\prime \prime \prime}-H a\left(f^{\prime}-\beta f f^{\prime \prime}\right)$,

$M\left(\frac{1}{2} \eta \phi^{\prime}+2 \phi\right)+f^{\prime} \phi-f \phi^{\prime}=\frac{1}{S c} \phi^{\prime \prime}-\gamma \phi$,

where $M=\frac{\alpha}{b}$ is the unsteadiness parameter, $\beta=b \lambda_{0}$ is the Maxwell parameter, $H a=\frac{\sigma B_{0}^{2}}{\rho b}$ is the magnetic parameter, $S c=\frac{v}{D}$ is the Schmidt number, $\gamma=\frac{k_{0}}{b}$ is the reaction rate parameter. Here $\gamma>0$ represents the destructive reaction, $\gamma=0$ corresponds to no reaction, and $\gamma<0$ stands for the generative reaction. The primes above denote differentiation with respect to $\eta$ only.

The boundary conditions (5) and (6) then become

$f^{\prime}=1, f=0, \phi=1$ at $\eta=0$

and $\quad f^{\prime} \rightarrow 0, \phi \rightarrow 0$ as $\eta \rightarrow \infty$.

\section{Numerical Method for Solution}

Equations (7) and (8) along with the boundary conditions (9) and (10) are solved by first converting to an initial value problem. We set

$$
\begin{aligned}
f^{\prime}=z, z^{\prime} & =p, \phi^{\prime}=q, p^{\prime}=M\left(\frac{\eta}{2} p+z\right)+z^{2}-f p+\beta\left(f^{2} p-2 f z p\right)+H a(z-\beta f p), \\
q^{\prime} & =S c\left\{(z \phi-f q)+\gamma \phi+M\left(\frac{\eta}{2} q+2 \phi\right)\right\} .
\end{aligned}
$$

with the boundary conditions

$$
f(0)=0, \quad f^{\prime}(0)=1, \quad \phi(0)=1 .
$$

In order to integrate (11) and (12) as an initial value problem we require a value for $p(0)$ and $q(0)$, i.e. $f^{\prime \prime}(0)$ and $\phi^{\prime}(0)$. But no such values are given at the boundary. The most important factor of shooting method is to choose the appropriate finite values for $\eta_{\infty}$. In order to determine $\eta_{\infty}$, we start with some initial guess value for some particular set of physical parameters and obtain values for $f^{\prime \prime}(0)$ as well as $\phi^{\prime}(0)$. The selection of $\eta_{\infty}$ is repeated until two successive values of $f^{\prime \prime}(0)$ and $\phi^{\prime}(0)$ differ only by a specified significant digit. The last value of $\eta_{\infty}$ is finally chosen to be the appropriate value of $\eta_{\infty}$ for that particular set of parameters. The value of $\eta_{\infty}$ may different for another set of physical parameters. Once the finite value of $\eta_{\infty}$ is determined then the integration is carried out. We compare the calculated values for $f^{\prime}$ and $\phi$ at $\eta=10$ (say) with the given boundary conditions $f^{\prime}(10)=0$ and $\phi(10)=0$ and adjust the estimated values, $f^{\prime \prime}(0)$ and $\phi^{\prime}(0)$, to give a better approximation for the solution.

We take series of values for $f^{\prime \prime}(0)$ and $\phi^{\prime}(0)$, and apply the fourth order classical Runge-Kutta method with step-size $\mathrm{h}=0.01$. The above procedure is repeated until we get the results up to the desired accuracy, with an error of $10^{-5}$ (Mukhopadhyay (2009)].

\section{Results and Discussion}

In order to validate the method used in this study and to judge the accuracy of the present analysis, a comparison with available results corresponding to the skin-friction coefficient $f^{\prime \prime}(0)$ for unsteady flow of viscous 

incompressible Newtonian fluid ( $\beta=0$ ) are compared with the available results of Sharidan et al. (2006) and Chamkha et al. (2010) in Table-1 and found that the results agree well.

Table-1: The values of $f^{\prime \prime}(0)$ for various values of unsteadiness parameter $M$ with $\beta=0$.

\begin{tabular}{|c|c|c|c|}
\hline $\mathrm{M}$ & Sharidan et al. (2006) & Chamakha et al. (2010) & Present study \\
\hline 0.8 & -1.261042 & -1.261512 & -1.261479 \\
\hline 1.2 & -1.377722 & -1.378052 & -1.377850 \\
\hline
\end{tabular}

In order to study the behavior of velocity and concentration fields for Maxwell fluid, a comprehensive numerical computation is carried out for various values of the parameters that describe the flow characteristics, and the results are reported in terms of graphs as shown in Fig.2-7. The ranges of the governing parameters which are considered in this study are as follows:

Unsteadiness parameter $M: 0$ to 0.3 , Maxwell parameter $\beta: 0$ to 0.4 , Magnetic parameter $H a: 0$ to 0.3 , Schmidt number Sc: 0.5 to 1 , reaction rate parameter $\gamma:-0.3$ to 0.3 .

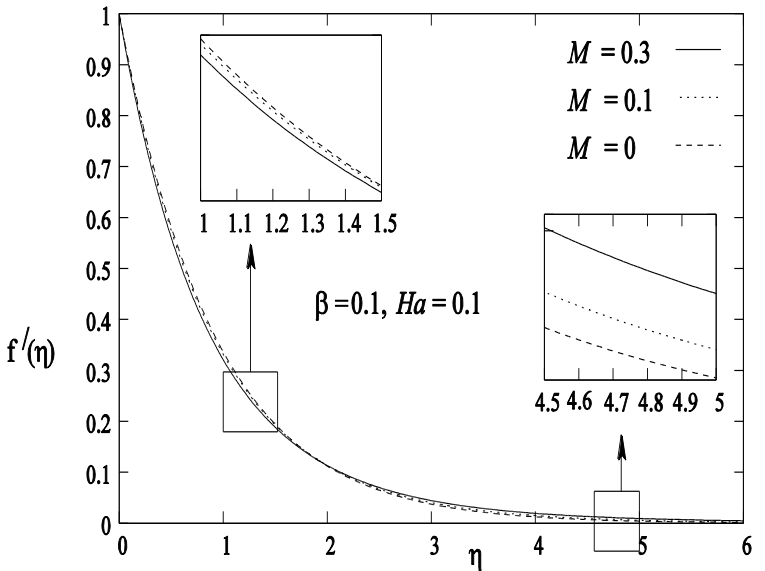

Fig. 2: (a) Velocity profiles for variable unsteadiness parameter M

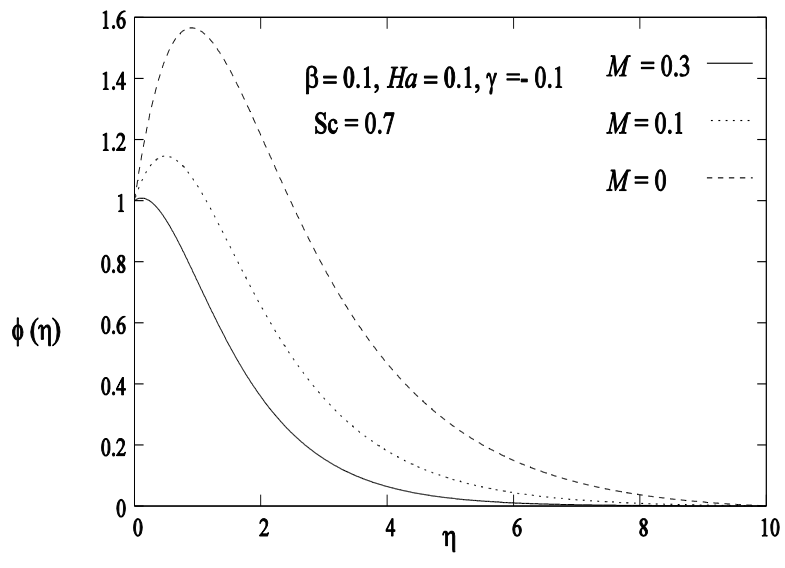

Fig. 2: (b) Concentration profiles for variable unsteadiness parameter $\mathrm{M}$.

Fig. 2(a) exhibits the velocity profiles for several values of unsteadiness parameter $M$. It is seen that the velocity along the sheet decreases initially with the increase of unsteadiness parameter $M$ and this implies an accompanying reduction of the thickness of the momentum boundary layer near the wall but away from the wall fluid velocity increases with increasing unsteadiness. $M=0$ indicates the steady case. Fig. 2(b) represents the effects of unsteadiness parameter on the solute distribution. From this figure, it is noticed that the concentration at a particular point is found to decrease significantly with increasing unsteadiness parameter. As the unsteadiness parameter $M$ increases, less mass is transferred from the fluid to the sheet; hence, the concentration $\phi(\eta)$ decreases [Fig.2(b)]. Rate of mass transfer (from the fluid to the sheet) decreases with increasing $M$ [see also Fig.7(b)]. Since the fluid flow is caused solely by the stretching sheet and the sheet surface concentration is higher than free stream concentration, the velocity and concentration decrease with increasing $\eta$. Concentration overshoot is noted. The concentration gradient at the wall is positive i.e. there is a mass transfer from the fluid to the sheet i.e. the mass absorption occurs. That is why the overshoot in the concentration profiles is noted near the surface.

Effects of Maxwell parameter $\beta$ on velocity and concentration profiles for unsteady motion are clearly exhibited in Fig. 3(a) \& (b) respectively. Here $\beta=0$ gives the result for viscous incompressible fluid. The effect of increasing values of $\beta$ is to reduce the velocity and hence the boundary layer thickness decreases [Fig. 3(a)] 

because with the increase of Maxwell parameter $\beta$ the intrinsic fluidity of a material becomes less. The velocity curves in Fig. 3(a) show that the rate of transport is considerably reduced with the increase of $\beta$.

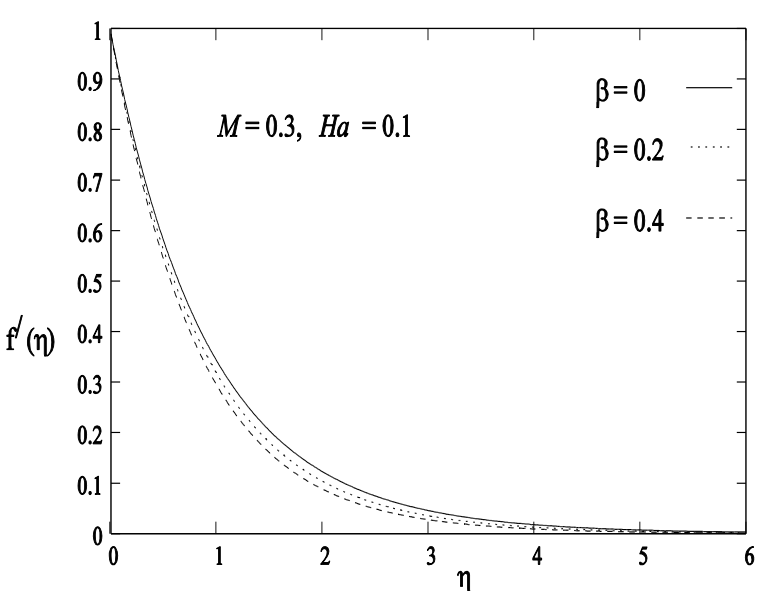

Fig. 3: (a) Velocity profiles for variable Maxwell parameter $\beta$ for unsteady motion

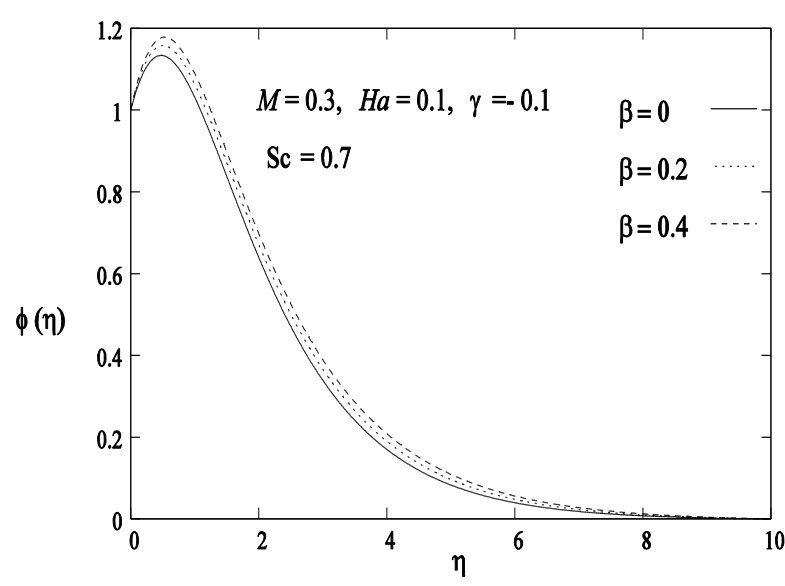

Fig. 3: (b) Concentration profiles for variable Maxwell parameter $\beta$ for unsteady motion

The effect of increasing $\beta$ leads to enhance the concentration field in unsteady motion [Fig.3(b)]. The thickening of the solute boundary layer occurs due to increase in the elasticity stress parameter. It can also be seen from Fig.3(a) that the momentum boundary layer thickness decreases as $\beta$ increases, and hence induces an increase in the absolute value of the velocity gradient at the surface. The value of the concentration gradient at the surface increases with an increase in $\beta$, as shown in Fig.3(b). Thus, the mass transfer rate at the surface increases with increase in $\beta$. The concentration field $\phi$ also decreases for large values of $\beta$ in case of generative chemical reaction $(\gamma<0)$. But the magnitude of $\phi$ is larger in case of $(\gamma<0)$ when compared with the case of destructive chemical reaction $(\gamma>0)$. It is also found that the concentration field is decreased for several values of $\beta$ in all cases $(\gamma=0, \gamma>0$ and $\gamma<0)$.

The effects of magnetic parameter $\mathrm{Ha}$ on velocity and concentration fields are exhibited in Fig.4(a) and Fig.4(b) respectively. With increasing magnetic field, fluid velocity decreases [Fig.4(a)] but the concentration increases in this case. The transverse magnetic field, applied normal to the flow direction, has a tendency to create a dragging force known as Lorentz force which opposes the motion of the fluid.

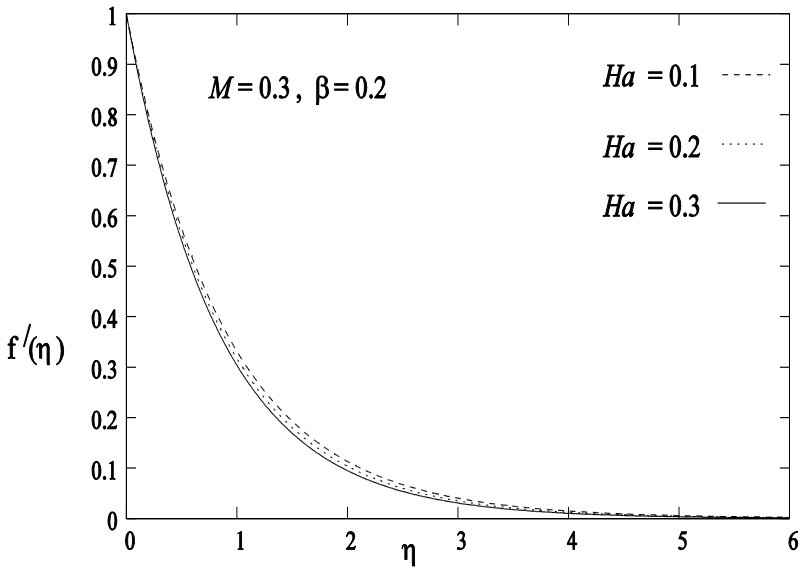

Fig. 4: (a) Velocity profiles for variable magnetic parameter, $\mathrm{Ha}$

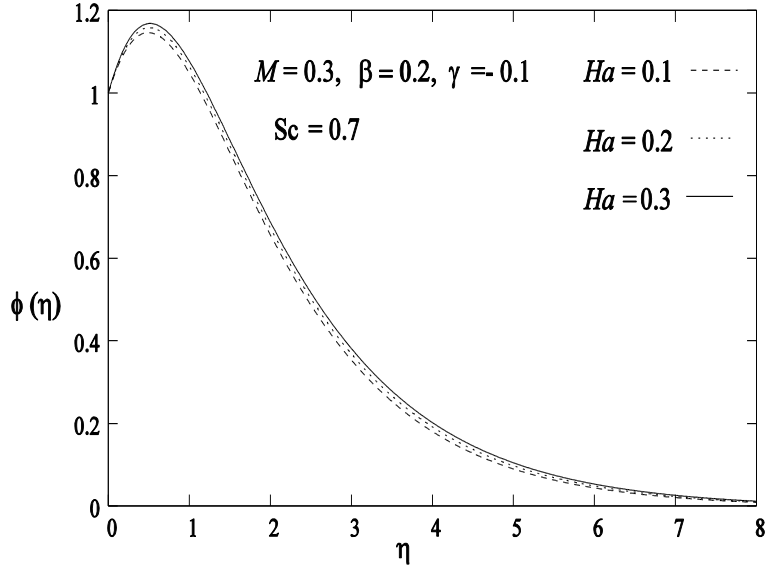

Fig. 4: (b) Concentration profiles for variable magnetic parameter, $\mathrm{Ha}$. 
Fig.5(a) illustrates the effects of reaction rate parameter $\gamma$ on concentration profiles. Concentration increases with the increase of reaction rate parameter $\gamma$. Concentration overshoot is noted for all values of $\gamma$ considered in this study [Fig.5(a)]. It is seen that concentration field increases for generative chemical reaction $(\gamma<0)$ where as it decreases for destructive chemical reaction $(\gamma>0)$. The reaction-rate parameter is a decelerating agent when $\gamma>0$. Note that the change in case of generative chemical reaction $(\gamma<0)$ is larger in comparison to the case of destructive chemical reaction $(\gamma>0)$. The concentration boundary layer decreases in case of destructive chemical reaction. This is because the conversion of the species takes place as a result of chemical reaction and thereby reduces the concentration in the boundary-layer.

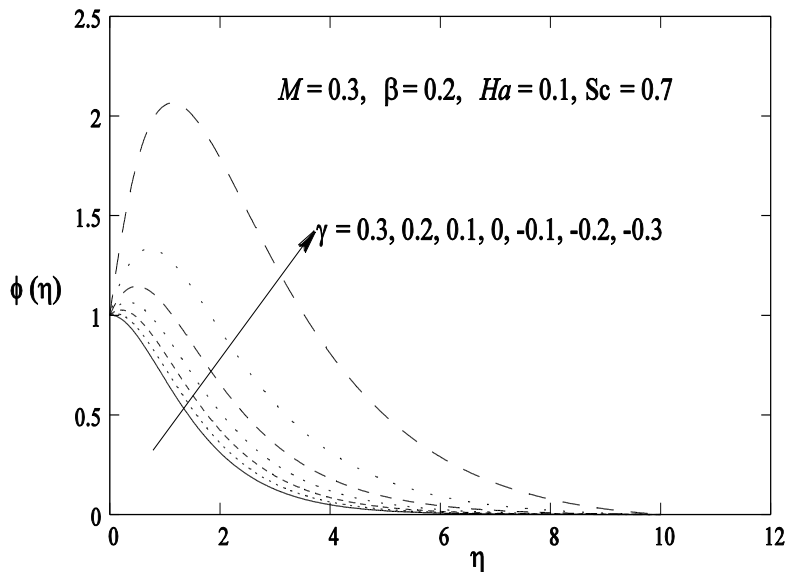

Fig. 5: (a) Concentration profiles for variable values of reaction rate parameter $\gamma$

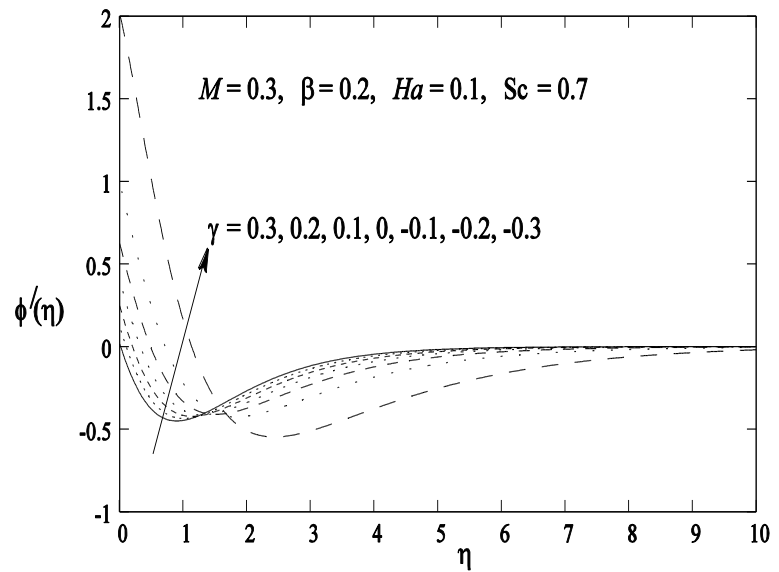

Fig. 5: (b) Concentration gradient profiles for variable values of reaction rate parameter $\gamma$

Fig.5(b) presents the behaviour of concentration gradient profiles for reaction rate parameter $\gamma$.It is found that initially (near the wall) concentration gradient increases for generative chemical reaction $(\gamma<0)$ where as it decreases for destructive chemical reaction $(\gamma>0)$ but far away from the wall opposite behaviour is noted [Fig.5(b)]. Hence, initially solute boundary layer thickness reduces with destructive chemical reaction $(\gamma>0)$ and becomes thicker for constructive chemical reaction $(\gamma<0)$. Actually, chemical reaction causes to increase the rate of interfacial mass transfer. The reaction reduces the local concentration, thus increasing its concentration gradient and its flux.

An interesting behavior of the concentration profiles for the variation of Schmidt number can be found from Fig.6. It is noted that initially (near the wall) the concentration increases but away from the wall it decreases with increasing Sc [Fig.6]. Moreover, the solute boundary layer thickness decreases by increasing Schmidt number. The Schmidt number is inversely proportional to the diffusion coefficient (D) and thus an increase in the values of $\mathrm{Sc}$ results in a decrease of diffusion coefficient. Consequently, with increase of diffusion coefficient, the concentration and concentration boundary layer thickness increase initially but due to unsteadiness concentration decreases finally.

Furthermore, the effects of unsteadiness parameter $M$ and Maxwell parameter $\beta$ on $\left[f^{\prime \prime}(0)\right]$ related to skinfriction coefficient and also the mass transfer coefficient are presented in Fig.7(a) and Fig.7(b) respectively. Value of $\left[f^{\prime \prime}(0)\right]$ related to skin-friction coefficient decreases with increasing unsteadiness parameter $M$ and also with Maxwell parameter $\beta$, but mass transfer rate at the surface $\left[\phi^{\prime}(0)\right]$ increases for $\beta$, decreases with 
S. Mukhopadhyay, R. S. R. Gorla/ Journal of Naval Architecture and Marine Engineering 9(2012) 123-133 $M$. A drop in skin friction as investigated in this paper has an important implication that in free coating operations, elastic properties of the coating formulations may be beneficial for the whole process. This means that less force maybe needed to pull a moving sheet at a given withdrawal velocity or equivalently higher withdrawal speeds can be achieved for a given driving force resulting an increase in the rate of production (Rajagopal (1995)].

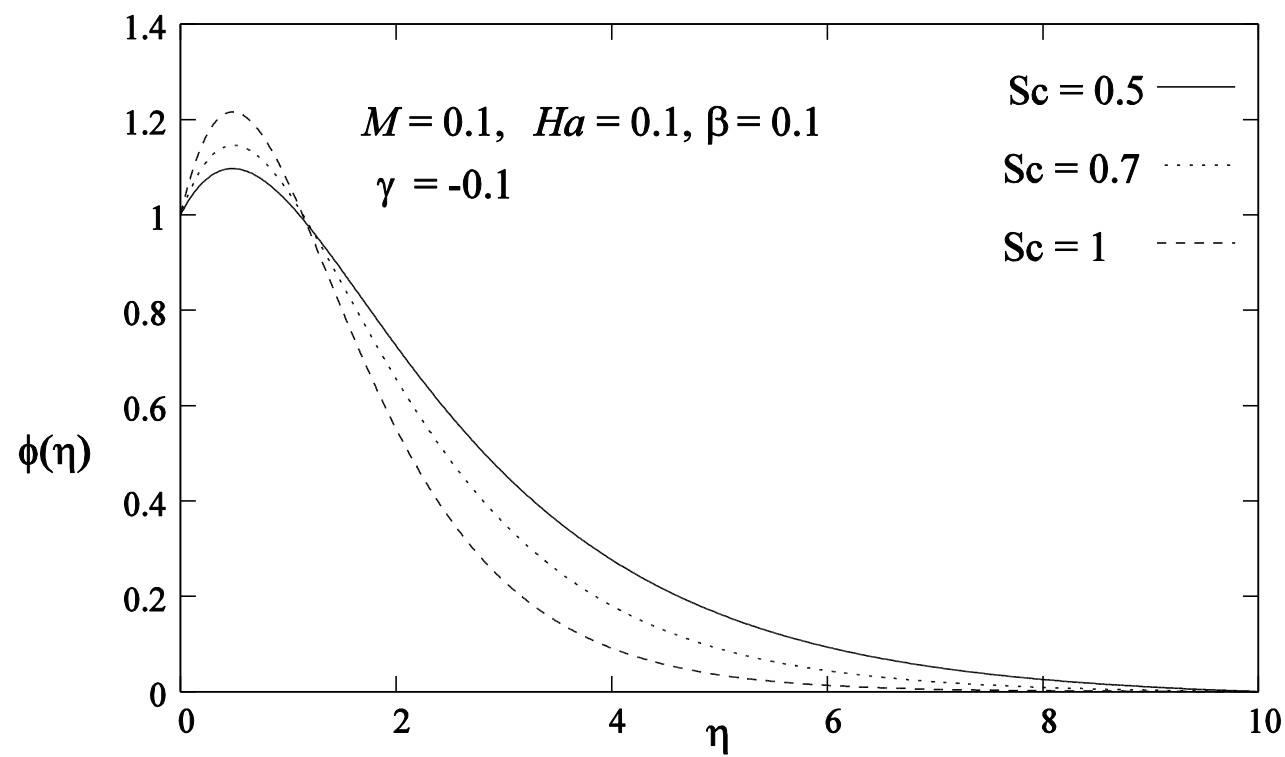

Fig.6: Concentration profiles for variable values of Schmidt number Sc.

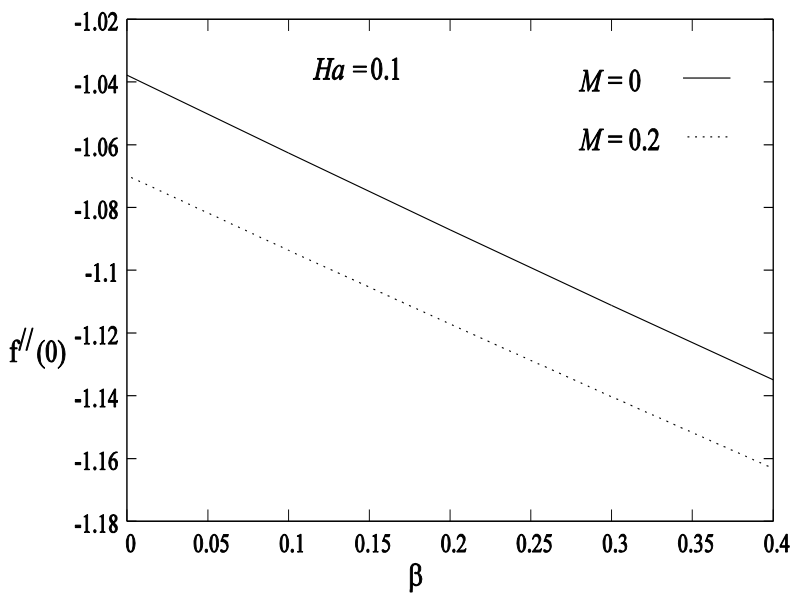

Fig. 7: (a) Variation of skin-friction coefficient with Maxwell parameter $\beta$ for two values of unsteadiness

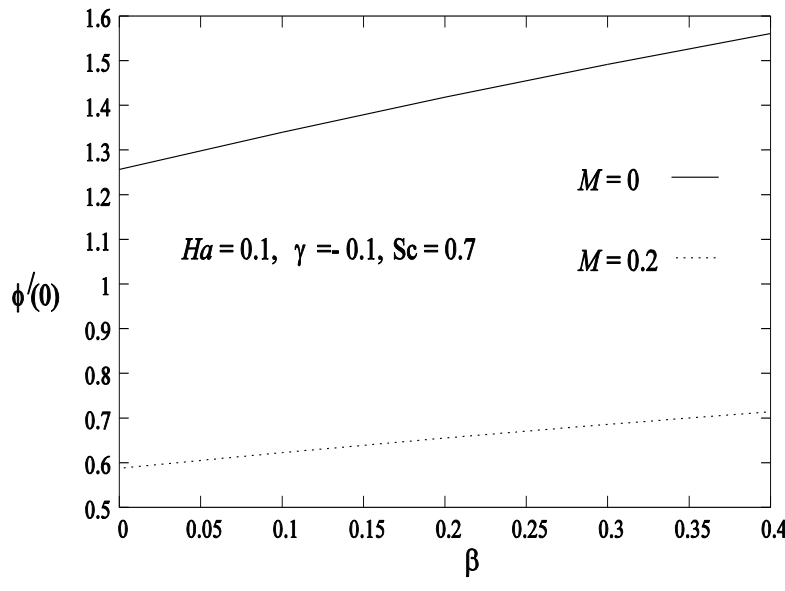

Fig.7: (b) Variation of mass transfer coefficient with Maxwell parameter $\beta$ for two values of unsteadiness parameter $M$

\section{Conclusions}

Similarity solutions for unsteady MHD boundary layer flow of a Maxwell fluid and mass transfer over a stretching surface in presence of first order constructive/destructive chemical reaction are obtained. It is found that fluid velocity decreases initially due to increase in unsteadiness parameter. The concentration also decreases significantly in this case. Also, it is found that the fluid velocity decreases with increasing magnetic parameter. The effect of increasing values of the Maxwell parameter is to suppress the velocity field. The concentration is enhanced with increasing Maxwell parameter. Mass transfer rate at the surface decreases with unsteadiness. Moreover, an increase in the Schmidt number reduces the solute boundary-layer thickness. The solute boundary- 
S. Mukhopadhyay, R. S. R. Gorla/ Journal of Naval Architecture and Marine Engineering 9(2012) 123-133 layer decreases in case of destructive chemical reaction. For a given Schmidt number, it is observed that the concentration increases during a generative chemical reaction and decreases in a destructive chemical reaction.

\section{References}

Abel, M. S., Sanjayan, E. and Nandeppanavar, M. M., (2008): Viscoelastic MHD flow and heat transfer over a stretchingsheet with viscous and ohmic dissipations, Commun. Nonlinear Sci. Numer. Simul., Vol.13, pp.18081821.

Ali, M. E. and Magyari, E., (2007): Unsteady fluid and heat flow induced by a submerged stretching surface while its steady motion is slowed down gradually, Int. J. of Heat and Mass Transfer, Vol.50 (1-2), pp.188-195, http://dx.doi.org/10.1016/j.ijheatmasstransfer.2006.06.021.

Andersson, H. I., Hansen, O. R. and Holmedal, B., (1994): Diffusion of a chemically reactive species from a stretching sheet, Int. J. Heat Mass Transfer, Vol. 37, pp.659-664.

Andersson, H. I., Aarseth, J. B. and Dandapat, B. S., (2000): Heat transfer in a liquid film on an unsteady stretching surface, Int. J. of Heat and Mass Transfer, Vol.43, pp.69-74, http://dx.doi.org/10.1016/S00179310(99)00123-4.

Bhattacharyya, K., Mukhopadhyay, S. and Layek, G.C., (2013):Unsteady MHD boundary layer flow with diffusion and first order chemical reaction over a permeable stretching sheet with suction or blowing, Chem. Engng. Commun., Vol.200, pp.1-19, http://dx.doi.org/10.1080/00986445.2012.712577

Chamkha, A. J., Aly, A. M. and Mansour, M. A., (2010): Similarity solution for unsteady heat and mass transfer from a stretching surface embedded in a porous medium with suction/injection and chemical reaction effects, Chem. Eng. Commun., Vol.197, pp. 846-858, http://dx.doi.org/10.1080/00986440903359087.

Cortell, R., (2007): Toward an understanding of the motion and mass transfer with chemically reactive species for two classes of viscoelastic fluid over a porous stretching sheet, Chem. Engng. Processing: Process Intensification, Vol.46, pp. 982-989.

Dandapat, B. S., Santra, B. and Andersson, H. I., (2003): Thermocapillarity in a liquid film on an unsteady stretching surface, Int. J. of Heat and Mass Transfer, Vol.46 (16), pp. 3009-3015, http://dx.doi.org/10.1016/S0017-9310(03)00078-4.

Dandapat, B. S., Santra, B. and Vajravelu, K., (2007): The effects of variable fluid properties and thermocapillarity on the flow of a thin film on an unsteady stretching sheet, Int. J. of Heat and Mass Transfer, Vol.50 (5-6), pp.991-996, http://dx.doi.org/10.1016/j.ijheatmasstransfer.2006.08.007.

Elbashbeshy, E. M. A. and Bazid, M. A. A., (2004): Heat transfer over an unsteady stretching surface, Heat Mass Transfer, Vol.41, pp.1-4, http://dx.doi.org/10.1007/s00231-004-0520-x.

Haroun, M. H., (2007): Effect of Deborah number and phase difference on peristaltic transport of a third-order fluid in an asymmetric channel, Commun.Nonlinear Sci. Numer. Simul., Vol.12, pp.1464-1480.

Hassanien, I. A., (1996): Flow and heat transfer on a continuous flat surface moving in a parallel free stream of power-law fluid, Appl.Model., Vol. 20, pp.779-783, http://dx.doi.org/10.1016/0307-904X(96)00082-0.

Hayat, T., Abbas, Z. and Sajid, M., (2006): Series solution for the upper-convected Maxwell fluid over a porous stretching plate, Phys. Lett. A, Vol.358, pp.396-403.

Hayat, T., Fetecau, C. and Sajid, M., (2008): On MHD transient flow of a Maxwell fluid in a porous medium and rotating frame, Phys. Lett. A, Vol.372, pp.1639-1644.

Hayat, T., Awais, M., Qasim, M., Hendi, A. A., (2011): Effects of mass transfer on the stagnation point flow of an upper-convected Maxwell (UCM) fluid, Int. J. of Heat and Mass Transfer, Vol. 54, pp.3777-3782.

Hayat, T., Shehzad, S. A. and Alsaedi, A., (2012a): Soret and Dufour effects on magnetohydrodynamic (MHD) flow of Casson fluid, Appl. Math. Mech. -Engl. Ed., Vol.33(10), pp.1301-1312.

Hayat, T., Iqbal, Z., Mustafa, M. and Alsaedi, A., (2012b): Momentum and heat transfer of an upper-convected Maxwell fluid over a moving surface with convective boundary conditions, Nuclear Engng. and Design, Vol. 252 , pp. 242- 247.

Heyhat, M. M. and Khabazi, N., (2010): Non-isothermal flow of Maxwell fluids above fixed flat plates under the influence of a transverse magnetic field, Proc. IMechE Part C: J. Mech. Eng. Sci., Vol. 225, DOI: 10.1243/09544062JMES2245.

Mukhopadhyay, S., (2009): Effect of thermal radiation on unsteady mixed convection flow and heat transfer over a porous stretching surface in porous medium, Int. J. Heat Mass Transfer, Vol.52, pp.3261-3265, http://dx.doi.org/10.1016/j.ijheatmasstransfer.2008.12.029 .

Mukhopadhyay, S., (2011): Heat transfer analysis for unsteady MHD flow past a non-isothermal stretching surface, Nuclear Engng and Design, Vol.241, pp.4835-4839. 
S. Mukhopadhyay, R. S. R. Gorla/ Journal of Naval Architecture and Marine Engineering 9(2012) 123-133 Mukhopadhyay, S. and Bhattacharyya, K., (2012: Unsteady flow of a Maxwell fluid over a stretching surface in presence of chemical reaction, J. of the Egyptian Math. Society (in press), http://dx.doi.org/10.1016/j.joems.2012.08.019.

Mukhopadhyay, S., (2012a): Heat transfer analysis for unsteady flow of a Maxwell fluid over a stretching surface in the presence of a heat source/sink, Chinese Phy. Letters, Vol. 29 (5), 054703.

Mukhopadhyay, S., (2012b): Upper-convected Maxwell fluid flow over an unsteady stretching surface embedded in porous medium subjected to suction/blowing, Z. Naturforsch., Vol.67a, pp.641 - 646, DOI: 10.5560/ZNA.2012-0075

Mukhopadhyay, S., (2012c): MHD Boundary layer slip flow along a stretching cylinder, Ain Shams Engng. J. (in press), DOI 10.1016/j.asej.2012.07.003.

Mukhopadhyay, S., (2012d): Slip effects on MHD boundary layer flow over an exponentially stretching sheet with suction/blowing and thermal radiation, Ain Shams Engng.J.,DOI: 10.1016/j.asej.2012.10.007.

Pahlavan, A. A. and Sadeghy, K., (2009): On the use of homotopy analysis method for solving unsteady MHD flow of Maxwellian fluids above impulsively stretching sheets, Commun. Nonlinear Sci. Numer. Simulat., Vol.14, pp.1355-1365, http://dx.doi.org/10.1016/j.cnsns.2008.03.001.

Rajgopal, K. R., (1995): Boundary layers in non-Newtonian fluids (Ed-s: M.D.P.

Montiero Marques, J.F. Rodrigues).

Sadeghy, K. and Sharifi, M., (2004): Local similarity solution for the flow of a 'second-grade' viscoelastic fluid above a moving plate, Int. J. Non-linear Mech., Vol.39, 1265-1273.

Sajid, M., Ahmad, I., Hayat, T. and Ayub, M., (2009): Unsteady flow and heat transfer of a second grade fluid over a stretching sheet, Commun. Nonlinear Sci. Numer. Simul., Vol.14, pp. 96-108, http://dx.doi.org/10.1016/j.cnsns.2007.07.014.

Serdar, B. and Salih Dokuz, M., (2006): Three-dimensional stagnation point flow of a second grade fluid towards a moving plate, Int. J. Engng Sci., Vol.44, pp.49-58.

Sharidan, S., Mahmood, T. and Pop, I., (2006): Similarity solutions for the unsteady boundary layer flow and heat transfer due to a stretching sheet, Int. J. Appl. Mech. Engng., Vol.11, pp.647-654.

Siddiqui, A. M., Zeb, A., Ghori, Q. K. and Benharbit, A. M., (2008): Homotopy perturbation method for heat transfer flow of a third grade fluid between parallel plates, Chaos Solitons Fractals, Vol.36, pp.182-192.

Tsai, R., Huang, K. H., Huang, J. S., (2008): Flow and heat transfer over an unsteady stretching surface with a non-uniform heat source, Int. Commun. Heat Mass Transfer, Vol. 35, pp.1340-1343, http://dx.doi.org/10.1016/j.icheatmasstransfer.2008.07.001.

Turkyilmazoglu, M. and Pop, I., (2013): Exact analytical solutions for the flow and heat transfer near the stagnation point on a stretching/shrinking sheet in a Jeffrey fluid, Int. J. of Heat and Mass Transfer, Vol.57, pp. 82-88, http://dx.doi.org/10.1016/j.ijheatmasstransfer.2012.10.006. 